\title{
New secrets behind bone metastasis
}

\author{
Xing Guo ${ }^{1}$, Xiao-Fan Wang ${ }^{2}$ \\ ${ }^{1}$ Department of Pharmacology, University of California, San Diego, La Jolla, CA 92093, USA; 2 Department of Pharmacology and \\ Cancer Biology, Duke University Medical Center, Durham, NC 27710, USA \\ Cell Research (2012) 22:1309-1311. doi:10.1038/cr.2012.99; published online 26 June 2012
}

Secreted proteins play essential roles in every step of cancer metastasis, while the identities and functions of those that contribute to tissue-specific metastasis are largely uncharacterized. Two articles in Cell Research report the discovery and functional analyses of novel secreted proteins that are biologically and clinically relevant to bone metastasis. The combinatory approaches represented here, together with advances in related technology, will promise a better understanding of the cancer secretome.

Cancer metastasis is a multi-step process during which the cancer cells need to constantly communicate with surrounding tissues and adapt to new environments. The communication between cancer cells and stroma is mediated by cell-surface proteins (such as receptors and adhesion molecules) and a slew of secreted substances including small compounds, cytokines, growth factors, extracellular matrix (ECM) proteins, enzymes as well as their modulators. Interactions among these components physically and chemically reshape the extracellular space and alter the signaling and gene expressions within tumor and stromal cells, which in turn cause further changes of the microenvironment, eventually leading to the establishment

Correspondence: Xing Guo ${ }^{\mathrm{a}}$, Xiao-Fan Wang ${ }^{\mathrm{b}}$

aE-mail: x4guo@ucsd.edu

bE-mail: xiao.fan.wang@duke.edu of a metastatic niche that supports the malignancy in a distal organ [1]. Cancer cells en route to metastasis display a different pattern of secretion than that from normal or non-metastatic cells, and secreted proteins can have a remarkable impact on the target organ tropism, e.g., metastasis to bone or lung [2-5].

Bone is a favorite metastatic site of several types of cancer. The intimate connection with the circulation system, richness in growth factors and ECM proteins, and entailment of multiple cell types all make the bone a highly conducive place for the thriving of invaded cancer cells [6]. In addition, bone tissues undergo frequent remodeling, which is regulated by numerous secreted proteases and is accompanied by high-volume flow of ions such as calcium, magnesium and phosphate. This also makes the bone a unique niche for tumor cells as compared to other target organs. Cancer patients with bone metastasis are often inflicted with severe pain and several related complications, and show poor responses to regular therapies. A number of therapeutic approaches have been devised to intervene this devastating process, most of which target secreted proteins or signals through their receptors [7]. Given the biological and clinical importance, it is absolutely necessary and rewarding to generate a systematic view of the cancer "secretome" that mediates bone metastasis.

Recent advances in mass spectrometry (MS) technology have allowed researchers to globally survey secreted proteins from in vitro (using conditioned medium of cultured cancer cells) and in vivo sources (from bodily fluids extracted near tumor tissues) [3]. However, a common problem associated with many of such "-omic" experiments is the accumulation of huge amounts of data without rigorous functional validation or clear biological insights. In addition, the secretome studies often face a particular criticism that intracellular proteins released by apoptotic or disrupted cells may give rise to false-positive results. Moreover, previous work on cancer secretomes has never been specifically aimed at bone metastasis. To address these issues, researchers from two laboratories have made valuable attempts $[8$, 9]. As discussed below, both groups have adopted a multi-layered approach consisting of: (1) MS detection of secreted proteins from conditioned media $(\mathrm{CM})$ of cancer cells with distinct potentials of bone metastasis, (2) bioinformatic sifting of MS data to eliminate non-secreted intracellular proteins, (3) comparison of secretome data with microarray data and clinical records, (4) validation of the expression of selected novel proteins in cancer cell lines, and (5) functional characterization of the identified proteins in vitro and in vivo.

Blanco et al. [8] performed both non-quantitative and quantitative MS analyses on secretomes of 8 cell lines derived from human breast cancer, human bladder cancer and mouse breast 
cancer. Among several hundred secreted proteins detected, dozens of them were found to be positively correlated with high probability of bone metastasis. These particular proteins were designated as BMSSs ("bone metastasis secretome signatures"). Functionally, they fall into several categories including growth factor signaling, ECM binding, peptidase regulation and calcium binding. All these activities regulate tumor-bone interactions and were highly represented in all bone-metastatic cells, regardless of tumor types and species. However, the individual BMSS proteins that carry out these functions show little overlap between human and mouse cells or between breast and bladder cancer, suggesting diverse molecular mechanisms underlying the secretome transformation. This observation is reminiscent of the vastly different alterations of oncogenes and tumor suppressors found in different tumors, which in the end almost always impinge on a few critical pathways that give rise to the common hallmarks of cancer [10]. Knowing this feature of the BMSSs will not only help us understand the pathogenesis of bone metastasis but also provide a guideline for diagnosis and individualized treatment of patients.

Blanco et al. [8] further examined three groups of newly identified BMSS candidates: COL6A1 and PLOD2 (collagen functionality proteins), PLAT-PLAU (plasminogen activators) and CST1-2-4 (peptidase inhibitors of the cystatin family). Although individual knockdown of each gene in MDA-MB-231 cells did not block bone metastasis in a xenograft model, combined expression of genes in the same functional group, e.g., CST1, 2 and 4, did significantly increased tumor burden in the bone. The authors also noted that clinical evidence seems to support co-overexpression of these functionally related genes. Therefore, the results suggest that both functional redundancy and synergism exist among these secreted proteins, and how exactly they each contribute to bone metastasis remains an open question.

Jin and Zhang et al. [9] performed a similar secretome study focusing on subclones from the MDA-MB-231 cells [4], and they also identified a positive correlation between bone metastasis and overproduction of CST1, 2 and 4. Moreover, they noticed that a related family member, CST6, was markedly less secreted in those highly bone-metastatic cells. Indeed, CST6 has broad inhibitory effects on cell proliferation, migration and invasion in vitro and shows tumor-suppressive activity in vivo. This is in sharp contrast with CST1, 2 and 4. Biochemical data hinted that these pro- and anti-metastasis cystatins may inhibit different proteases [9]. Shutting down CST6 while allowing for CST1, 2, 4 expression apparently can be advantageous to metastatic tumor cells, which is again reminiscent of the gain/loss of function of canonical oncogenes/tumor suppressors. It is curious to know the target proteases of different cystatins in vivo and their functions in breast cancer bone metastasis, as well as how the selective inhibition is achieved. In a broader sense, the extracellular "degradome" controlled by secreted proteases and their modulators is an interesting subject worth exploring using pharmacological tools and genetic models. In years to come, we can anticipate more mechanistic studies that will bring forth profound insights to the roles of secreted protein in metastasis.

An important aspect of both these studies is cross-referencing the secretome data with those from transcriptome studies and clinical samples. With the enormous data generated using diverse systems and techniques, multiplexing of different "-omes" has become increasingly important not only because it offers a great way for data validation and quality control, but also because it provides us with a holistic perspective of the biology, helps to reveal multi-level regulations and points out new directions. For instance, both groups indicate that changes in protein secretion do not always result from altered transcription of the coding genes. This is not unexpected as secreted proteins undergo extensive post-translational modifications (PTM), such as phosphorylation, glycosylation, proteolysis, sulfation, acetylation, etc. These PTMs to a great extent determine the amount, quality and activity of the secreted proteins. A very recent example is the discovery of a Golgi-localized kinase named Fam20C, which phosphorylates a good number of secreted proteins involved in calcium binding and biomineralization [11]. Surprisingly, Fam20C itself is a secreted kinase. It would be of great importance to elucidate whether and how regulators of the secretory pathway (such as Fam20C) participate in the process of bone metastasis, as they represent another class of therapeutic targets.

How else are the secreted proteins regulated in metastatic cancer cells? The CST6 gene is epigenetically silenced by promoter methylation [9]. Several secreted factors that promote breast cancer bone metastasis can be transcriptionally upregulated by growth factors such as TGF- $\beta$ [4]. Since breast cancer and prostate cancer can both spread to bone, and these two types of cancer show remarkable similarities such as their responses to steroid hormones [12], could they produce and/or respond to common BMSS proteins? How does the secretome evolve as cancer cells proceed through the different phases of metastasis? How does the secretome change in response to anti-cancer therapies? Do cancer stem cells have a unique secretory pattern? Answers may not be readily available for all of these questions, and yet decoding the secretory signatures of metastatic cancers will enable early detection of the disease as well as more accurate prediction of clinical outcomes.

One caveat for interpreting the results shown in these two papers is that in vitro cultured cancer cells cannot reflect the heterogeneous nature of real tumors, and ingredients of conditioned media do not fully represent the complexity of 
the tumor microenvironment. The cancer secretome comprises products from both cancer and stromal cells, and the secreted proteins can function in autocrine, paracrine or even endocrine manners. Therefore, a fundamental question regarding the BMSS proteins is their in vivo cell origin and targets. Jin and Zhang et al. [9] showed that CST6 is mostly expressed in the epithelial compartment of tissue sections, which is consistent with its cell-autonomous roles in vitro. One commonly used method to mimic tumorstroma interaction is cell co-culture, such as with cancer-associated fibroblasts or tumor-associated macrophages, in which the secreted proteins can be genetically manipulated. However, the best system to address the above question would be animals with tissue-specific knockout of any particular secreted protein. In addition, in vivo sampling techniques such as laser capture microdissection and capillary ultrafiltration probes have been used in conjunction with MS analysis [3]. These will be powerful tools for studying the "real" cancer secretome.

Finally, we should realize that the cancer secretome is more than secreted proteins. Small signaling molecules, metabolites and RNAs can all be secreted and found in the milieu of a tumor. These non-protein molecules also play an essential part in tumor biology and should not be neglected. Significant efforts have been and will still be dedicated to finding sensitive and reliable biomarkers as well as drug targets from the cancer secretome. In this regard, the two publications discussed here are informative and encouraging. Despite the biological and technical challenges, we envisage a productive future of secretome studies with more secrets behind cancer metastasis to be uncovered.

\section{References}

1 Psaila B, Lyden D. The metastatic niche: adapting the foreign soil. Nat Rev Cancer 2009; 9:285-293.

2 Nguyen DX, Bos PD, Massague J. Metastasis: from dissemination to organspecific colonization. Nat Rev Cancer 2009; 9:274-284.

3 Karagiannis GS, Pavlou MP, Diamandis EP. Cancer secretomics reveal pathophysiological pathways in cancer molecular oncology. Mol Oncol 2010; 4:496-510.

4 Kang Y, Siegel PM, Shu W, et al. A multigenic program mediating breast cancer metastasis to bone. Cancer Cell 2003; 3:537-549.
5 Minn AJ, Gupta GP, Siegel PM, et al. Genes that mediate breast cancer metastasis to lung. Nature 2005; 436:518524.

6 Weilbaecher KN, Guise TA, McCauley LK. Cancer to bone: a fatal attraction. Nat Rev Cancer 2011; 11:411-425.

7 Mundy GR. Metastasis to bone: causes, consequences and therapeutic opportunities. Nat Rev Cancer 2002; 2:584593.

8 Blanco M, LeRoy G, Khan Z, et al. Global secretome analysis identifies novel mediators of bone metastasis. Cell Res 2012; 22:1339-1355.

9 Jin L, Zhang Y, Li H, et al. Differential secretome analysis reveals CST6 as a suppressor of breast cancer bone metastasis. Cell Res 2012; 22:1356-1373.

10 Hanahan D, Weinberg RA. Hallmarks of cancer: the next generation. Cell 2011; 144:646-674.

11 Tagliabracci VS, Engel JL, Wen J, et al. Secreted kinase phosphorylates extracellular proteins that regulate biomineralization. Science 2012; 336:11501153.

12 Risbridger GP, Davis ID, Birrell SN, Tilley WD. Breast and prostate cancer: more similar than different. Nat Rev Cancer 2010; 10:205-212. 